\title{
Immunohistochemical expression of Olig2, CD99, and EMA to differentiate oligodendroglial-like neoplasms
}

\author{
Maher Kurdi ${ }^{1}$, Charles Eberhart ${ }^{2}$ \\ ${ }^{1}$ Department of Pathology, Faculty of Medicine in Rabigh, King Abdulaziz University, Saudi Arabia, ${ }^{2}$ Department of Pathology, \\ the John Hopkins University School of Medicine, Baltimore, United States
}

\begin{abstract}
Aim of the study: This study has assessed the diagnostic ability of oligodendrocyte-2 (Olig2), CD99, and epithelial membrane antigen (EMA) immunohistochemical stains to diagnose oligodendroglial-like neoplasms as central neurocytoma, ependymoma, or oligodendroglioma.

Material and methods: An immunohistochemistry (IHC) panel of Olig2, EMA, and CD99 was performed on 18 central neurocytomas, 46 ependymomas, and 28 oligodendrogliomas. A quantitative labelling index of stained tumor cells was assessed using a scoring system, and its diagnostic predictability was evaluated with multinomial logistic regression. Results: Significant differences in IHC expression patterns were observed between all tumor groups $(p<0.001)$. The labeling indices of the histochemical expression of Olig2, EMA, and CD99 were related to diagnostic predictability. Olig2 was unlikely to differentiate ependymoma from central neurocytoma $(p=0.154)$, while EMA and CD99 were significant in diagnosing these two tumors $(p<0.05)$. Olig2 was a specific marker of oligodendroglioma, differentiating it from ependymoma and central neurocytoma $(p<0.001)$. EMA and CD99 were unlikely to differentiate oligodendroglioma from central neurocytoma $(p>0.05)$, but CD99 significantly differentiated ependymoma from oligodendroglioma $(p=0.022)$. These labelling indices were used to re-assess the diagnostic accuracy, regardless of tumor location and histology, and yielded significantly different tumor diagnoses.

Conclusions: The IHC panel of Olig2, EMA, and CD99 should be used to differentiate oligodendroglial-like neoplasms. Olig2 is a specific IHC marker to diagnose oligodendroglioma and differentiate it from ependymoma and central neurocytoma. Lack of Olig2 expression rules out oligodendroglioma and suggests the diagnosis of ependymoma rather than central neurocytoma if the EMA labelling index shows diffuse/partial expression. CD99 is considered a sensitive marker for ependymoma but not central neurocytoma.
\end{abstract}

Key words: oligodendroglial-like neoplasm, CD99, EMA, Olig2, immunohistochemistry.

\section{Introduction}

Oligodendroglial-like neoplasms of the central nervous system include oligodendroglioma (grade II/III), ependymoma (grade II/III), and central neurocytoma (grade II/III). They share common cytomorphological features (e.g., monomorphism, indeterminate clear cell component, and minimal vascularity) but with different histogenesis. The only way to differentiate between 
them is by immunohistochemical staining of certain markers, including oligodendrocyte-2 (Olig2), CD99, and epithelial membrane antigen (EMA). Olig2, a member of the group of basic helix-loop-helix transcription factors [7], is commonly expressed in oligodendroglial tumors $[4,11]$ but its expression in ependymoma remains poorly elucidated $[5,6,9]$. The lack of its expression does not only rule out oligodendroglioma, but Olig2 staining is considered potentially more reliable than EMA staining for the diagnosis of ependymoma [5]. Although EMA is of little value for differentiating between all ependymoma subtypes [8], it is more selective than CD99 in differentiating between grade II and III ependymomas [10]. CD99, the product of the MIC2 gene, is a cell surface antigen expressed in several tumors [1]. CD99 has also been reported to aid in distinguishing between oligodendroglioma and ependymoma and in diagnosing clear cell brain tumors through the visualization of CD99-negative clear cells [5]. Owing to the diagnostic dilemma in differentiating these tumors, our current study has attempted to assess the ability of Olig2, EMA, and CD99 to differentiate oligodendroglial-like neoplasms (i.e., oligodendroglioma, ependymoma, central neurocytoma), particularly those with clear cell components. The histochemical expression of tumor cells has been quantitatively assessed through the labelling index-based scoring system.

In this study, we aimed to assess the diagnostic ability of Olig2, CD99, and epithelial EMA immunohistochemical stains to distinguish oligodendroglial-like neoplasms as central neurocytoma, ependymoma, or oligodendroglioma.

\section{Material and methods Patient stratification}

This study included 92 patients with oligodendroglial-like neoplasms [central neurocytomas $(n=18)$, ependymomas ( $n=46)$, and oligodendrogliomas $(n=28)$ ] from two medical institutions in Saudi Arabia between 2017 and 2020. The study was ethically approved by the National Biomedical Ethics Committee at King Abdulaziz University (HA-02-J-008) (reference number 178-19). The clinical patient data retrieved from the hospital records included age, gender, and tumor type and location. All the patients were stratified into three groups on the basis of the histological tumor subtype.

\section{Methodology}

Four unstained sections of formalin-fixed paraffin embedded samples of each tumor were prepared for hematoxylin and eosin staining and immunohistochemistry (IHC) of Olig2, EMA, and CD99 (Figs. 1-2).

\section{IHC staining for CD99, EMA, and Olig2}

Immunohistochemistry assays on formalin-fixed paraffin embedded tissue sections were performed with the ultraView DAB detection kit on a BenchMark XT automated staining system (Ventana). The following rabbit/mouse monoclonal antibodies were used at concentrations of 1 : 200: anti-CD99 (013), anti-EMA (E29), and anti-Olig2 (EPR2673, Abcam, ab109186, United States). The standard procedure included deparaffinization with EZ Prep at $75^{\circ} \mathrm{C}$, heat pre-treatment in Cell Conditioning solution (CC1; Ventana) for $60 \mathrm{~min}$, and primary incubation for $1 \mathrm{~h}$ at $37^{\circ} \mathrm{C}$.

Results: For CD99 and EMA, dot-like membranous staining on tumor cell membranes was defined as positive expression. Ependymomas exhibiting diffuse membranous staining were also considered positive. For Olig2, nuclear and perinuclear staining of tumor cells was considered positive expression (Figs. 2-3). This parameter was correlated with the study by Choi et al. [1].
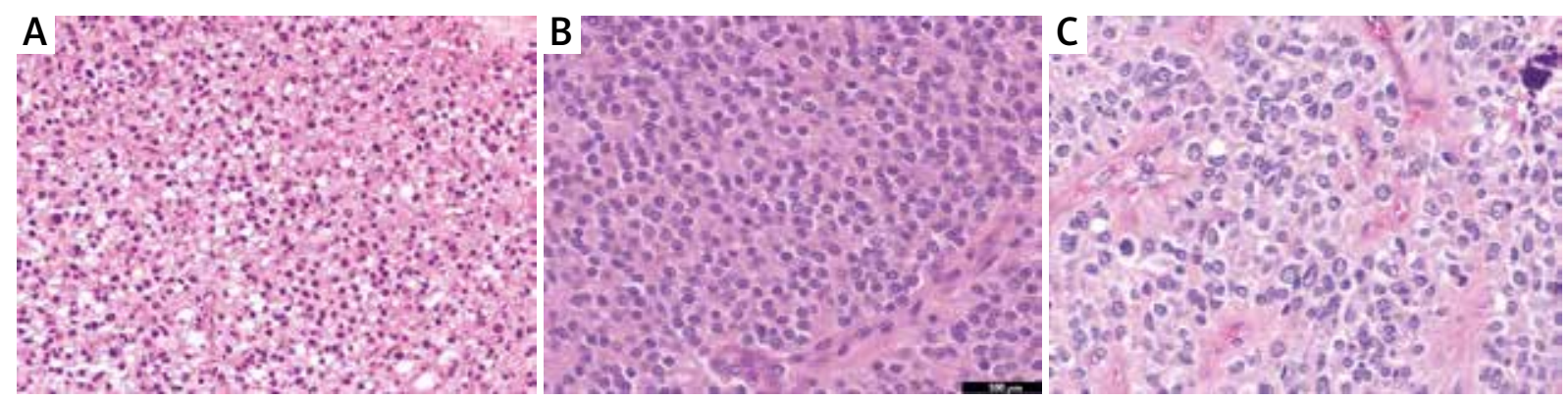

Fig. 1. Oligodendroglial-like neoplasms. A) Oligodendroglioma, B) central neurocytoma, and C) ependymoma with clear cell morphology. Magnification: 100x. 

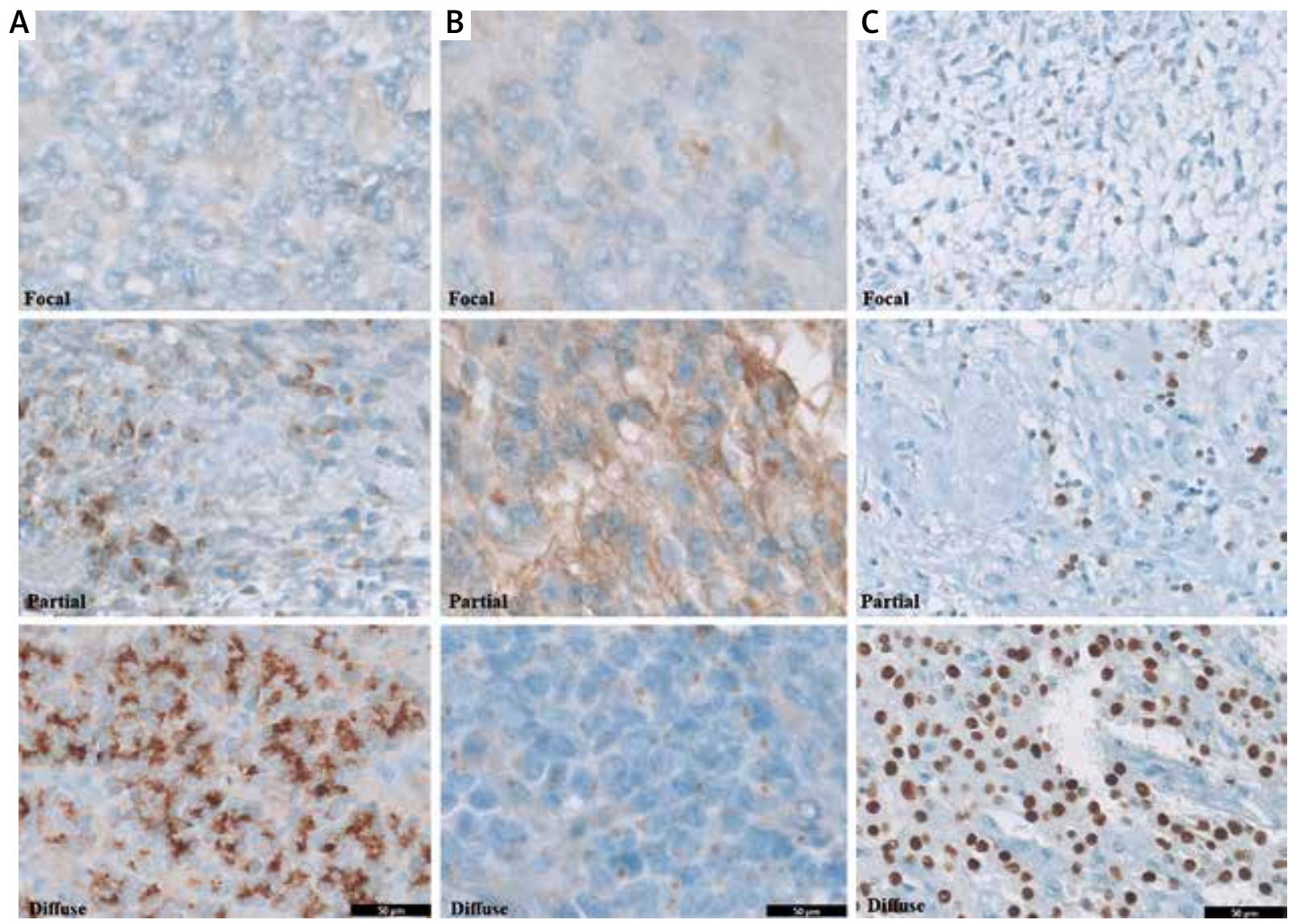

Fig. 2. Immunohistochemistry pattern of A) EMA, B) CD99, and C) Olig2 expression by using the quantitative labelling index. Magnification: 50x.
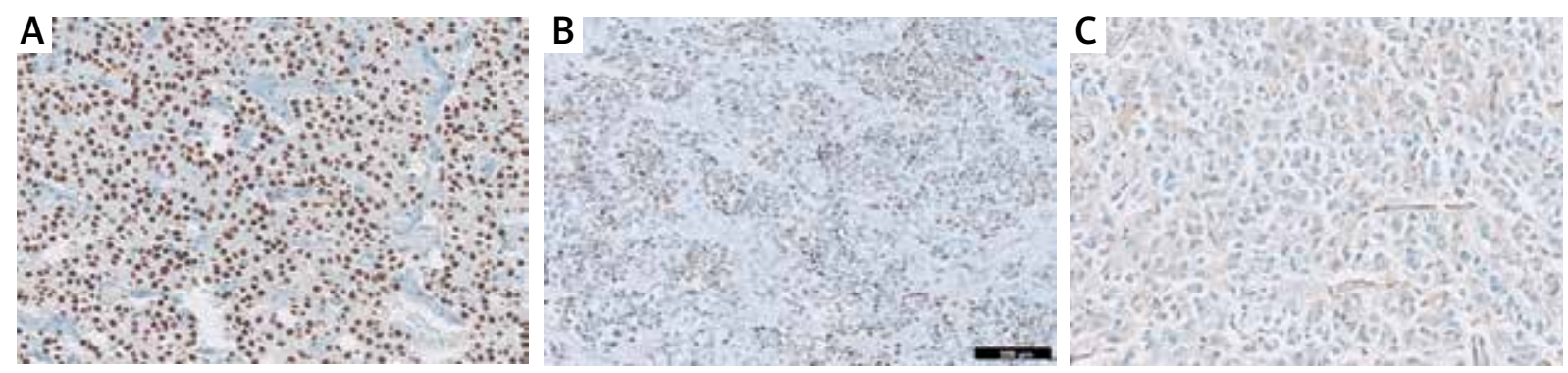

Fig. 3. Unusual histochemical expression in some oligodendroglial-like neoplasms. A) Olig2 expression in central neurocytoma, B) Olig2 expression in ependymoma, C) CD99 expression in oligodendroglioma. Magnification: $100 \times$.

\section{Quantitative analysis of Olig2, EMA, CD99 histochemical expression on oligodendrogliomas, ependymomas, and central neurocytomas using light microscopy}

After immunostaining, a focal random area of solid representative tumor of positive expression was evaluated under light microscopy using high-power (40x) magnification. Tumor cells were counted manually by two neuropathologists, and the average was calculated. The labelling index was quantitatively assessed using the following equation: Labelling index $=[($ Staining-positive nuclei) $) /($ Staining-positive nuclei + Staining-negative nuclei] (Table I). 


\section{Statistical methods}

The data were described as frequencies and percentages used the Fisher's Exact test. The clustered simple error bar means plot with $95 \%$ confidence interval $(\mathrm{Cl})$ was used to visualize the comparison of the labelling indices, and multinomial logistic regression analysis was used to test the diagnostic predictability of the three oligodendroglial-like neoplasms. A $p$ value of $<0.05$ was considered statistically significant. All statistical analyses in this study were performed using IBM SPSS1 statistical software program ver. 24 (SPSS Inc., Chicago, IL).

\section{Results}

This study included 92 patients with different oligodendroglial-like neoplasms [mean age: $21 \pm 4$ years; 53 men (58\%) and 39 women (42\%)]. Tumors were divided into 18 central neurocytomas, 46 ependymomas, and 28 oligodendrogliomas.

\section{Statistical analysis}

Table II clearly describes the quantitative scores of the labelling indices for each IHC marker (Olig2, EMA, and CD99) in each tumor group based on the scoring system used. There was a significant difference in the pattern of IHC expression between the tumor
Table I. The labelling index (\%) was assessed through the following scoring system

\begin{tabular}{|lc|}
\hline Expression & Labelling index (\%) \\
\hline No expression & 0 \\
\hline Focal expression & $>0-20$ \\
\hline Partial expression & $>20-50$ \\
\hline Diffuse expression & $>50$ \\
\hline
\end{tabular}

For statistical analysis, the scores were divided by 100.

groups ( $p<0.001)$. For example, Olig2 showed significant diffuse expression in oligodendrogliomas (96.4\%, $p<0.001)$ compared to ependymomas and central neurocytomas, while EMA and CD99 expression was low or absent in oligodendrogliomas ( $0 \%$ and $7.1 \%$, respectively). EMA also showed focal/partial expression in ependymomas (26-50\%). Focal EMA expression was observed in 4 (22.2\%) out of 18 (100\%) central neurocytomas. CD99 showed no expression in $53.6 \%$ of oligodendrogliomas but showed diffuse/partial expression in 37-45\% of ependymomas. CD99 was expressed focally in $55.6 \%$ of central neurocytomas. Moreover, unusual expression was detected in the tumor groups (Fig. 4). Unusual diffuse Olig2 expression was detected in $5(27.8 \%)$ out of $18(100 \%)$ central neurocytomas, and focal expression was detected in 19 (41.3\%) out of 46 (100\%) ependymomas. CD99 was minimally expressed in some cases of oligodendroglioma.

Table II. Quantitative analysis of labelling indices for each immunohistochemistry marker between oligodendroglial-like neoplasms

\begin{tabular}{|c|c|c|c|c|c|}
\hline & $\begin{array}{l}\text { Central neurocytoma } \\
\qquad(n=18)\end{array}$ & $\begin{array}{l}\text { Ependymoma } \\
\qquad(n=46)\end{array}$ & $\begin{array}{l}\text { Oligodendroglioma } \\
\qquad(n=28)\end{array}$ & Total $(n=92)$ & $P$ value \\
\hline Olig2 expression & & & & & $<0.001^{1}$ \\
\hline Absent & $7.0(38.9 \%)$ & $13.0(28.3 \%)$ & $0.0(0.0 \%)$ & $20.0(21.7 \%)$ & \\
\hline Focal expression & $6.0(33.3 \%)$ & $19.0(41.3 \%)$ & $0.0(0.0 \%)$ & $25.0(27.2 \%)$ & \\
\hline Partial expression & $0.0(0.0 \%)$ & $12.0(26.1 \%)$ & $1.0(3.6 \%)$ & $13.0(14.1 \%)$ & \\
\hline Diffuse expression & $5.0(27.8 \%)$ & $2.0(4.3 \%)$ & $27.0(96.4 \%)$ & $34.0(37.0 \%)$ & \\
\hline EMA expression & & & & & $<0.001^{1}$ \\
\hline Absent & $14.0(77.8 \%)$ & $9.0(19.6 \%)$ & $27.0(96.4 \%)$ & $50.0(54.3 \%)$ & \\
\hline Focal expression & $4.0(22.2 \%)$ & $23.0(50.0 \%)$ & $1.0(3.6 \%)$ & $28.0(30.4 \%)$ & \\
\hline Partial expression & $0.0(0.0 \%)$ & $12.0(26.1 \%)$ & $0.0(0.0 \%)$ & $12.0(13.0 \%)$ & \\
\hline Diffuse expression & $0.0(0.0 \%)$ & $2.0(4.3 \%)$ & $0.0(0.0 \%)$ & $2.0(2.2 \%)$ & \\
\hline CD99 expression & & & & & $<0.001^{1}$ \\
\hline Absent & $1.0(5.6 \%)$ & $2.0(4.3 \%)$ & $15.0(53.6 \%)$ & $18.0(19.6 \%)$ & \\
\hline Focal expression & $10.0(55.6 \%)$ & $6.0(13.0 \%)$ & $8.0(28.6 \%)$ & $24.0(26.1 \%)$ & \\
\hline Partial expression & $5.0(27.8 \%)$ & $21.0(45.7 \%)$ & $3.0(10.7 \%)$ & $29.0(31.5 \%)$ & \\
\hline Diffuse expression & $2.0(11.1 \%)$ & $17.0(37 \%)$ & $2.0(7.1 \%)$ & $31.0(22.8 \%)$ & \\
\hline
\end{tabular}

\footnotetext{
${ }^{1}$ Fisher's Exact test
} 
Table III. Diagnostic differentiation of oligodendroglial-like neoplasms using histochemical Olig2, EMA, and CD99 expression and the average labeling index-based scoring system

\begin{tabular}{|c|c|c|c|c|c|c|c|c|}
\hline \multicolumn{2}{|c|}{ Differential diagnosis } & \multicolumn{2}{|c|}{ Predictor } & Estimate & SE & $Z$ & $p$ & Odds ratio \\
\hline \multirow{4}{*}{\multicolumn{2}{|c|}{$\begin{array}{l}\text { Ependymoma - central } \\
\text { neurocytoma }\end{array}$}} & \multicolumn{2}{|c|}{ Intercept } & -1.11 & 0.634 & -1.749 & 0.081 & 0.3297 \\
\hline & & \multicolumn{2}{|c|}{ Olig2 labelling index } & -2.338 & 1.638 & -1.427 & 0.154 & 0.0965 \\
\hline & & \multicolumn{2}{|c|}{ EMA labelling index } & 26.79 & 8.867 & 3.021 & 0.003 & $4.31 \mathrm{E}+11$ \\
\hline & & \multicolumn{2}{|c|}{ CD99 labelling index } & 3.496 & 1.372 & 2.548 & 0.011 & 32.9713 \\
\hline \multirow{4}{*}{\multicolumn{2}{|c|}{$\begin{array}{l}\text { Oligodendroglioma - } \\
\text { central neurocytoma }\end{array}$}} & \multicolumn{2}{|c|}{ Intercept } & -2.41 & 1.15 & -2.095 & 0.036 & 0.0898 \\
\hline & & \multicolumn{2}{|c|}{ Olig2 labelling index } & 4.928 & 1.328 & 3.711 & $<0.001$ & 138.0401 \\
\hline & & \multicolumn{2}{|c|}{ EMA labelling index } & -90.617 & 88.589 & -1.023 & 0.306 & $4.42 \mathrm{E}-40$ \\
\hline & & \multicolumn{2}{|c|}{ CD99 labelling index } & -0.86 & 1.62 & -0.531 & 0.595 & 0.423 \\
\hline \multirow{4}{*}{\multicolumn{2}{|c|}{$\begin{array}{l}\text { Oligodendroglioma - } \\
\text { ependymoma }\end{array}$}} & \multicolumn{2}{|c|}{ Intercept } & -1.28 & 1.254 & -1.02 & 0.307 & 0.2776 \\
\hline & & \multicolumn{2}{|c|}{ Olig2 labelling index } & 7.4 & 2.034 & 3.64 & $<0.001$ & 1630.7061 \\
\hline & & \multicolumn{2}{|c|}{ EMA labelling index } & -141.06 & 91.426 & -1.54 & 0.123 & $5.48 \mathrm{E}-62$ \\
\hline & & \multicolumn{2}{|c|}{ CD99 labelling index } & -4.4 & 1.917 & -2.29 & 0.022 & 0.0123 \\
\hline \multicolumn{9}{|c|}{ Multinomial logistic regression. } \\
\hline & & & & \multicolumn{5}{|c|}{ Predicted diagnosis } \\
\hline & & & & Central $r$ & ocytoma & Ependyr & $\mathrm{ma}$ & Oligodendroglioma \\
\hline \multirow{6}{*}{$\begin{array}{l}\text { Differential } \\
\text { diagnosis }\end{array}$} & \multirow{2}{*}{\multicolumn{2}{|c|}{ Central neurocytoma }} & Count & & & 2 & & 4 \\
\hline & & & $\%$ & & & 11. & & 22.2 \\
\hline & \multirow{2}{*}{\multicolumn{2}{|c|}{ Ependymoma }} & Count & & & 40 & & 0 \\
\hline & & & $\%$ & & & 87. & & 0.0 \\
\hline & \multirow{2}{*}{\multicolumn{2}{|c|}{ Oligodendroglioma }} & Count & & & 2 & & 26 \\
\hline & & & $\%$ & & & 7.1 & & 92.9 \\
\hline
\end{tabular}

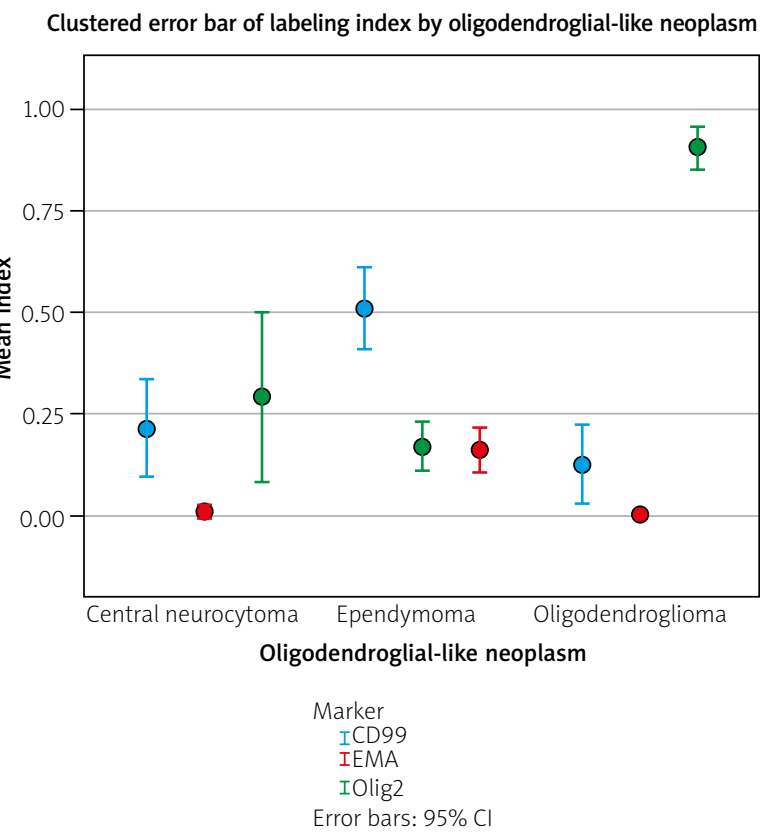

Fig. 4. The diagnostic predictability of oligodendroglial-like neoplasms using immunohistochemistry of Olig2, EMA, and CD99 and the labelling index-based scoring system.
Labeling indices of the histochemical expression of Olig2, EMA, and CD99 in oligodendroglial-like neoplasms showed significant effects on diagnostic predictability (Fig. 4, Table III). Olig2 was not efficient in differentiating ependymomas from central neurocytomas ( $p=0.154)$, while EMA and CD99 expression enabled differentiating between these two tumors ( $p=0.003$ and $p=0.011$, respectively). However, focal/partial EMA expression suggests the diagnosis of ependymoma rather than central neurocytoma. Conversely, Olig2 was a significant marker for differentiating oligodendrogliomas from ependymomas and central neurocytomas $(p<0.001)$. Furthermore, EMA and CD99 are unlikely to differentiate oligodendrogliomas from central neurocytomas as both rarely showed positive expression $(p>0.05)$, whereas the CD99 labelling index could significantly differentiate ependymomas from oligodendrogliomas $(p=0.022)$. From the model represented in Table III and Figure 2, the diagnostic accuracy has been re-assessed on the basis of the labelling indices of histochemical expression regardless of tumor location or histological variations. Around $66 \%$ of the 
cases diagnosed as central neurocytomas retained the same diagnosis, while $11 \%$ and $22 \%$ of the cases have been re-diagnosed as ependymomas or oligodendrogliomas, respectively. Around $87 \%$ of ependymoma cases retained the same diagnosis, while $13 \%$ of the cases have been re-diagnosed as central neurocytomas. Approximately $7 \%$ of the ependymoma cases, which showed clear cellular morphology, were misdiagnosed as oligodendroglioma before using the current labelling index-scoring system (Table III).

\section{Discussion}

Oligodendroglial-like neoplasms of the central nervous system include oligodendroglioma (grade II/III), ependymoma (grade II/II), and central neurocytoma (grade II/III). They share common cytomorphological features (e.g., monomorphism, indeterminate clear cell components, and minimal vascularity) but with different histogeneses. Thus, the only way to differentiate between them is with immunohistochemistry of Olig2, CD99, and EMA.

Olig2, a member of the group of basic helixloop-helix transcription factors, is essential for the development of neural progenitors and oligodendrocytes [7]. Several studies have confirmed prominent Olig2 expression in both oligodendroglial tumors and oligoastrocytomas [4,11]; however, its expression in glioblastomas and ependymomas remains poorly elucidated $[5,6,9]$. Although Olig2 expression was reported to be low to moderate in ependymomas [6], the applicability of Olig2 as a marker for the diagnosis of ependymomas remains undetermined. The lack of Olig2 expression does not only rule out oligodendrogliomas but is considered potentially more reliable than EMA for diagnosing ependymomas [5]. Hasselblatt et al. found that five punctate dots/high power fields of EMA staining in ependymomas was associated with a sensitivity of $72 \%$ and a specificity of $81 \%$ [3]. However, EMA had little value in differentiating between all subtypes of ependymoma [8] but exhibited more selective staining for differentiating between grade II and III ependymomas compared to CD99 [10]. CD99, the product of the MIC2 gene, is a cell surface antigen expressed in Ewing's sarcoma, choroid plexus papilloma, and primitive neuroectodermal tumors [1]. CD99 is also strongly expressed in ependymomas [1]. CD99 immunohisto- chemistry is also unique in that it is helpful in distinguishing between oligodendrogliomas and ependymomas as well as in the diagnosis of clear cell brain tumors through the visualization of CD99-negative clear cells [5]. Mahfouz et al. found that CD99 could further differentiate ependymal tumors from non-ependymal tumors [8]. These results were similar to the conclusions of Choi et al. and Fellinger et al. [1,2].

Our findings have revealed that Olig2 is a specific IHC marker to diagnose oligodendroglioma and differentiate it from ependymoma and central neurocytoma. The lack of Olig2 expression rules out oligodendroglioma and suggests ependymoma rather than central neurocytoma if EMA exhibits diffuse/ partial expression with the labelling index. CD99 can differentiate ependymoma from oligodendroglioma but not from central neurocytoma. Although Olig2 was expressed in rare cases of central neurocytoma, EMA was almost not expressed in cases of oligodendroglioma. This indicates that Olig2 together with EMA can be used to differentiate these two tumors.

The limitation acknowledged in our study is the lack of molecular study including $1 p 19 q$ deletion, however this study will be helpful at the centers where advanced molecular testings are absent and the diagnostic accuracy is only dependent on histomorphology and immunohistochemistry.

\section{Conclusions}

Immunohistochemistry of Olig2, EMA, and CD99 should be performed to differentiate cases of oligodendroglial-like neoplasms. The most specific diagnostic marker for oligodendroglioma is Olig2, as it showed high expression levels with the labelling index. EMA is considered a differentiating marker between ependymomas and central neurocytomas. CD99 is considered the most sensitive differentiating marker for ependymomas compared to other tumor groups, as it showed moderate-to-high expression levels. The diagnostic predictability of these stains in central neurocytomas is somewhat difficult to establish; however, focal expression of CD99 or Olig2 with absent EMA are probable findings.

\section{Availability of data and material}

The data that support the findings of this study are available from the corresponding author (MK) upon request. 


\section{Acknowledgments}

This project was funded by the Deanship of Scientific Research (DSR), at King Abdulaziz University, Jeddah, under grant no. J: 7-828-1440.

\section{Disclosure}

The authors report no conflict of interest.

\section{References}

1. Choi YL, Chi IG, Suh YL. CD99-immunoreactivity in ependymoma. Appl Itnmunohislochem Mol Morph 2001; 9: 125-129.

2. Fellinger EJ, Garin-Chesa P, Triche TJ, Huvos AG, Rettig WJ. Immunohistochemical analysis of Ewing's sarcoma cell surface antigen p30/32MIC2. Am J Pathol 1991; 139: 317-325.

3. Hasselblatt M, Paulus W. Sensitivity and specificity of epithelial membrane antigen staining patterns in ependymomas. Acta Neuropathol (Berlin) 2003; 4: 385-388.

4. Hoang-Xuan K, Aguirre-Cruz L, Mokhtari K, Marie Y, Sanson M. OLIG-1 and 2 gene expression and oligodendroglial tumours. Neuropathol Appl Neurobiol 2002; 28: 89-94.

5. Ishizawa K, Komori T, Shimada S, Hirose T. Olig2 and CD99 are useful negative markers for the diagnosis of brain tumours. Clin Neuropath 2008; 27: 118-128.

6. Ligon KL, Alberta JA, Kho AT, Weiss J. Kwaan MR, Nutt CL, Louis DN, Stiles CD, Rowitch DH. The oligodendroglial litieage marker OL1CJ2 is universally expressed in diffuse gliomas. J Neuropathol Exp Neuro 2004; 63: 499-509.

7. Lu QR, Yuk D, Alhena JA, Zhu Z, Pawlitzky L, Chan J, McMahon AP, Stiles CD, Rowitch DH. Sonic hedgehog-regulated oligodendrocyte lineage genes encoding bHLH proteins in the mammalian central nervous system. Neuron 2000; 25: 317-329.

8. Mahfouz S, Aziz AA, Gabel SM, el-Sheikh S. Immunihistochemical study of CD99 and EMA expression in ependymoma. Medscape J Med 2008; 10: 41.

9. Preusser M, Budka H, Rossler K, Hainfellner JA. Olig2 is a useful immunohistochemical marker in differential diagnosis of clear cell primary CNS neoplasms. Histopathology 2007; 50: 365-370.

10. Vege K, Giannini C, Scheithauer B. The immunophenotype of ependymomas. Appl Immunohistochem Mol Morphol 2000; 8: 25-31.

11. Yokoo H, Nobusawa S, Takebayashi H. Anti-human Olig2 antibody as a useful immunohistochemical marker of normal oligodendrocytes and gliomas. Am J Pathol 2004; 164: 1717-1725. 